\title{
Características clínicas y demográficas de pacientes con trauma raquimedular Experiencia de seis años
}

\author{
Clinical and demographic characteristics of \\ patients with spinal cord injury \\ Six years experience
}

\author{
Carlos Carvajal, Carlos Pacheco, Camilo Gómez-Rojo, \\ Julio Calderón, Carlos Cadavid, Fabián Jaimes • Medellín (Colombia)
}

\section{Resumen}

Introducción: el trauma raquimedular (TRM) es una entidad potencialmente catastrófica y la información acerca de las características del TRM penetrante relacionadas con violencia, particularmente las heridas por arma de fuego es escasa.

Diseño del estudio: estudio de cohorte retrospectiva.

Objetivos: determinar las características clínicas y demográficas de la población con TRM atendidas en un hospital de tercer nivel de Medellín.

Métodos: revisamos las historias clínicas de los pacientes admitidos con diagnóstico de TRM entre enero de 2005 y diciembre de 2010 . Se recolectaron datos con respecto a estancia hospitalaria y en la unidad de cuidados intensivos (UCI), el estado vital y la recuperación neurológica al egreso.

Resultados: tuvimos 68 casos de TRM con una frecuencia de 12 casos por 1000 pacientes admitidos a la UCI. La edad media fue de 28 años y $91 \%$ fueron del sexo masculino. La principal causa de TRM fue herida por arma de fuego (57\%) seguido por accidentes de tránsito (32\%). La estancia media de UCI fueron seis días, $56 \%$ de los pacientes requirió ventilación mecánica y 37\% requirió traqueostomía. El nivel de lesión medular más frecuente fue cervical. La mortalidad hospitalaria fue de $21 \%$, todos estaban con ventilación mecánica y entre más alto fue el nivel de lesión medular mayor era la mortalidad.

Conclusiones: nuestra investigación muestra que la población principalmente afectada por el TRM en nuestro medio son los jóvenes y la mortalidad, así como la estancia, complicaciones infecciosas y discapacidad tienen relación directa con el nivel de lesión medular. (Acta Med Colomb 2014; 40: 45-50).

Palabras clave: epidemiología, mortalidad, trauma raquimedular, trauma agudo, heridas por arma de fuego.

\footnotetext{
Abstract

Introduction: spinal cord injury (SCI) is a potentially catastrophic entity and information about the characteristics of pervasive SCI related to violence, particularly gunshot wounds, is scarce.

Study Design: Retrospective cohort study.

Objectives: to determine the clinical and demographic characteristics of the population with SCI attended in a tertiary hospital in Medellin.

Methods: The medical records of patients admitted with a diagnosis of SCI between January 2005 and December 2010 were reviewed. Data regarding stay in the hospital and intensive care unit (ICU), vital status and neurological recovery at hospital discharge were collected.

Results: there were 68 cases of SCI at a rate of 12 cases per 1000 patients admitted to the ICU. The average age was 28 years and $91 \%$ were male. The main cause of SCI was gunshot wounds (57\%) followed by traffic accidents (32\%). The mean ICU stay was six days, $56 \%$ of patients required mechanical ventilation and $37 \%$ required tracheostomy. The most common level of spinal
}

Dres. Carlos Carvajal, Dr. Carlos Pacheco, Camilo Gómez-Rojo, Julio Calderón y Carlos Cadavid: Departamento de Cuidado Crítico, Hospital Pablo Tobón Uribe; Dr. Fabián Jaimes: MSc, PhD Departamento de Cuidado Crítico. Unidad de Investigaciones, Hospital Pablo Tobón Uribe. Departamento de Medicina Interna, Universidad de Antioquia. Medellín (Colombia).

Correspondencia. Dr. Carlos Carvajal. Medellín (Antioquia).

E-mail: ccarvajal@hptu.org.co

Recibido: 6/II/2014 Aceptado: 23/II/2015 
cord injury was cervical. Hospital mortality was $21 \%$, all were mechanically ventilated and the higher the level of spinal cord injury, the greater was the mortality.

Conclusions: our research shows that people mainly affected by the SCI in our environment are young and mortality, as well as stay, infectious complications and disability are directly related to the level of spinal cord injury. (Acta Med Colomb 2014; 40: 45-50).

Keywords: epidemiology, mortality, spinal cord trauma, acute trauma, gunshot wounds.

\section{Introducción}

El trauma raquimedular (TRM) es una entidad potencialmente catastrófica, que aunque puede presentarse en individuos de cualquier edad suele afectar a personas de la segunda y tercera década de la vida, genera enormes costos médicos, psicosociales y económicos y tiene un impacto directo sobre el individuo, su familia y la sociedad (1).

La mortalidad asociada al TRM ha disminuido desde $70 \%$ en la Primera Guerra Mundial hasta menos de 5\% en la actualidad (2); no obstante la incidencia de lesiones medulares se ha incrementado en los últimos años desde 15 hasta 40 casos por millón de personas-año en el mundo (3, 4). De los 160000 casos nuevos por año que ocurren en los Estados Unidos, los pacientes del sexo masculino son los principalmente afectados en relación 4 a 1 con respecto a las mujeres $(1,5)$ y más de la mitad de estos casos se presentan en personas menores de 30 años $(6,7)$.

Las características clinicodemográgicas de los pacientes con TRM en el mundo han sido descritas ampliamente en diferentes trabajos de investigación. El nivel neurológico más frecuentemente comprometido es el cervical (16-75\%), seguido del compromiso torácico (16-36\%) y lumbar $(9-17 \%)(1,7)$. La principal causa son los accidentes de tránsito $(50 \%)$, seguidas por las caídas $(20 \%)$ y las lesiones deportivas $(9 \%)(1,6,8)$. Pese a que existe esta información, en países en vías de desarrollo las principales causas suelen diferir de las anteriormente mencionadas y aparecen mecanismos de trauma relacionados con la violencia. Entre éstos se encuentran las heridas por arma de fuego y objetos cortopunzantes, los cuales no han sido apropiadamente caracterizados en los trabajos de investigación.

La ventilación mecánica se ha convertido en el soporte básico de pacientes con TRM que desarrollan falla respiratoria secundaria (9-11). Del 50\% del total de los pacientes que ingresan a una UCI y requieren ventilación mecánica, $4 \%$ corresponde a pacientes con diagnóstico de TRM agudo $(9,12,13)$. Se sabe que casi $40 \%$ de los pacientes con lesión medular necesitarán ventilación mecánica con una duración media de 35-40 días y estos van a presentar una mayor incidencia de complicaciones respiratorias (traqueobronquitis, neumonías y atelectasias). Los pacientes más afectados por estas complicaciones van a ser aquellos que tengan lesiones cervicales con una prevalencia estimada de 40-70\% (14).

En los pacientes con TRM agudo, más aquellos con compromiso cervical, se esperan tiempos prolongados de ventilación mecánica con díficil desmonte de la misma, razón por la cual es frecuente la necesidad de realizar traqueostomía para favorecer el proceso de la liberación $(12,13,15,16)$. Esto está relacionado a la denervación del diafragma y de los músculos accesorios de la respiración y se presenta más frecuentemente con compromiso medular más alto. Por otro lado y aunque es claro que la implementación de protocolos para liberación del ventilador mecánico reducen la duración total de la ventilación, la necesidad de traqueostomía y el mejor método de desmonte en pacientes con TRM son desconocidos (11-13, 15).

Del tipo de TRM dependerán las secuelas neurológicas y la capacidad de recuperación del paciente. Se sabe que $30 \%$ de los pacientes que presentan TRM no penetrante van a tener recuperación neurológica completa (7) y 5\% de estos pacientes van a requerir soporte ventilatorio crónico $(8,12,13)$. Desafortunadamente no existe suficiente información con respecto a este tópico en TRM penetrante. Por esta razón, el objetivo de nuestro estudio fue evaluar las características clínicas y demográficas, así como también el proceso de recuperación, de los pacientes con TRM hospitalizados en nuestra institución entre 2005 y 2010.

\section{Métodos}

Locación: el Hospital Pablo Tobón Uribe es un hospital de tercer nivel de 365 camas ubicado en Medellín (Colombia) con dos unidades mixtas de cuidados intensivos con un total de 37 camas.

Diseño: estudio de cohorte retrospectiva.

Población: se incluyeron pacientes mayores de 16 años hospitalizados desde enero 1 de 2005 hasta diciembre 31 de 2010 con trauma raquimedular, de acuerdo con el diagnóstico de ingreso: CIE-10 S-141-146; S-240-246; S-340-348. Se excluyeron pacientes con fracturas raquídeas sin lesión neurológica y pacientes con información incompleta en la historia clínica.

Recolección de datos: el protocolo fue aprobado por el comité de investigación institucional. Por ser un estudio descriptivo retrospectivo en donde no se realizaron intervenciones y se tomaron los resultados de la historia clínica, no hubo necesidad de firmar consentimiento informado. La información se manejó en consolidado protegiendo la confidencialidad de los pacientes. Dos investigadores independientemente revisaron las historias clínicas y recolectaron las variables en un formato prediseñado.

Variables: se recolectó información con respecto a la 
edad, género, causas de trauma, trauma asociado, severidad de la enfermedad crítica con APACHE II (17), nivel neurológico, complicaciones infecciosas y no infecciosas, tratamiento (médico o quirúrgico), duración y características de la ventilación mecánica, método de desmonte de la ventilación mecánica y desenlace. Las causas del trauma fueron categorizadas en cuatro grupos: i) accidente de tránsito que incluía accidentes que ocurrían cuando el sujeto estaba dentro del vehículo o como peatón, ii) caídas, iii) lesiones deportivas y iv) violencia la cual incluía heridas por arma de fuego y por objetos cortopunzantes. El nivel neurológico y extensión de la lesión fue definido usando la definición de ASIA (American Spinal Injury Association) $(18,19)$. Este nivel de lesión fue definido como el segmento más caudal de la médula espinal con sensibilidad y función motora normal de forma bilateral.

Desenlaces: la estancia en UCI y hospitalaria. El estado vital, la recuperación y condición neurológica fueron evaluados al momento del alta. La recuperación neurológica fue categorizada como completa, incompleta y no recuperación. La condición neurológica fue establecida de acuerdo con la severidad (parálisis completa o incompleta) y patrón de lesión (hemi -para o cuadri-).

Análisis de datos: las variables nominales son presentadas como proporciones y las variables continuas como medias o medianas con rangos intercuartiles (RIC) y desviación estándar (SD), respectivamente. La supervivencia hospitalaria de acuerdo con el nivel de lesión neurológica está presentada por estimado de Kaplan-Meier. Todos los análisis fueron realizados con STATA/IC 12.1 (Stata Co. College Station, TX, USA).

\section{Resultados}

Durante el periodo de seis años de seguimiento hubo 5523 admisiones a la UCI y 68 casos de TRM. Ningún paciente fue excluido por información incompleta. Si se asume una tasa constante de ocurrencia, la frecuencia estimada es de 12 casos por 1000 pacientes admitidos a la UCI. La edad media de la población del estudio fue de 28 años (RIC $=22-39$ años) y $91 \%(n=62)$ correspondían a pacientes de sexo masculino. La Figura 1 muestra las causas de TRM en nuestra población. La violencia, específicamente lesiones por arma de fuego, fue la principal causa de TRM $(57 \%, \mathrm{n}=39)$ seguido por accidentes de tránsito $(32 \%, \mathrm{n}$

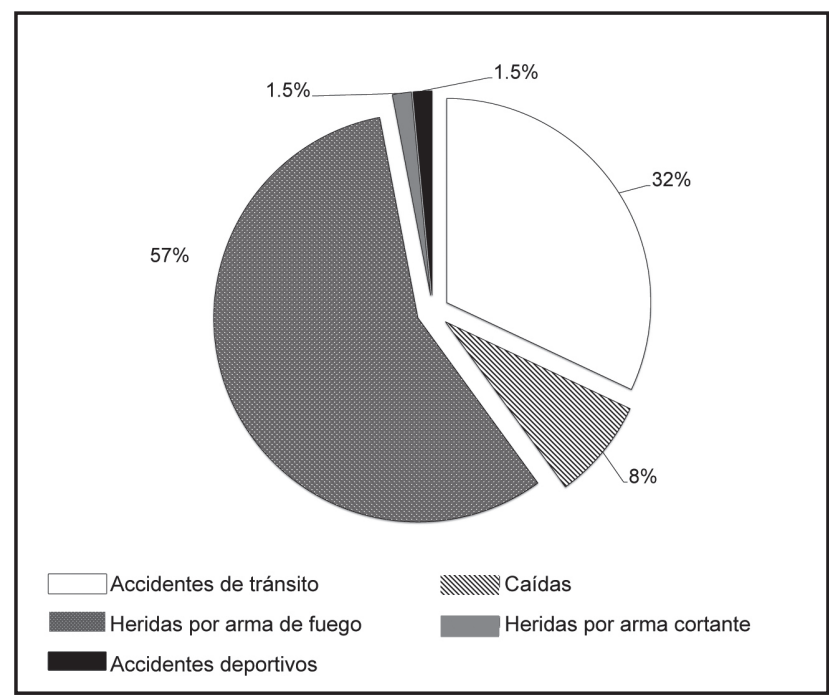

Figura 1. Causas de trauma raquimedular. Las principales causas de trauma raquimedular fueron la herida por proyectil de arma de fuego y el accidente de tránsito.

= 22). Todos los pacientes tuvieron una lesión asociada: trauma de tórax $(57 \%, \mathrm{n}=39)$, trauma en extremidades $(24 \%, \mathrm{n}=16)$, trauma encéfalocraneano $(21 \%, \mathrm{n}=14) \mathrm{y}$ trauma abdominal $(18 \%, \mathrm{n}=12)$. El APACHE II medio fue de $8(\mathrm{SD}=6.1)$ aunque a nueve pacientes $(13 \%)$ no se les calculó esta escala.

Un total de 44 pacientes $(65 \%)$ desarrollaron al menos una complicación, infecciosa $(\mathrm{n}=28 ; 41 \%)$ o no infecciosa ( $\mathrm{n}=40 ; 59 \%)$ y 24 pacientes presentaron ambas complicaciones $(35 \%)$. La más frecuente complicación no infecciosa fue el choque medular en 25 pacientes (37\%), seguido por atelectasias en 21 (31\%) y úlceras por presión en 8 (12\%). Entre las complicaciones infecciosas la neumonía fue la principal $(n=20,29 \%)$ seguida por infección del tracto urinario $(n=16,24 \%)$. La estancia media en la UCI fue de seis días con un rango de entre 1 y 103 días ( RIC = 2-20.5 días). 38 (56\%) pacientes requirieron ventilación mecánica y 14 de éstos (37\%) requirieron traqueostomía. Al 14\% (n= 2) se le realizó dentro de los primeros siete días. El nivel más frecuente de lesión fue el cervical $(47 \%, \mathrm{n}=32)$ y torácico $(47 \%, \mathrm{n}=32)$ seguido por lumbar $(6 \%, \mathrm{n}=4)$. A $27(38 \%)$ pacientes se les realizó fijación y descompresión quirúrgica. Algunas características clínicas y complicaciones, de acuerdo con el nivel de lesión, son comparadas en la Tabla 1.

Tabla 1. Características clínicas y complicaciones de acuerdo con el nivel de lesión en trauma raquimedular.

\begin{tabular}{|c|c|c|c|c|}
\hline & C3-C5 $(n=20)$ & C6-C7 $(n=12)$ & Tórax $(n=32)$ & Lumbar $(n=4)$ \\
\hline Puntaje APACHE II & $8.5(3-15,18)$ & $6(4-10,11)$ & $8(3-11,27)$ & $2(1-18,3)$ \\
\hline Tiempo de estancia hospitalaria (días) & $16(5-25)$ & $33(14-56.5)$ & $16.5(10.5-29)$ & $8(5.5-16)$ \\
\hline Ventilación mecánica & $13(65)$ & $9(75)$ & $15(47)$ & $1(25)$ \\
\hline Complicaciones infecciosas & $7(35)$ & $9(75)$ & $11(34)$ & $1(25)$ \\
\hline Complicaciones no infecciosas & $13(65)$ & $8(67)$ & $19(59)$ & 0 \\
\hline
\end{tabular}


No hubo un protocolo constante en el desmonte de la ventilación mecánica. Tres pacientes $(8 \%)$ fueron dados de alta con ventilación mecánica y $23(60 \%)$ fueron liberados exitosamente de ésta. La mortalidad total fue de $21 \%$ (n =14). Todos los pacientes que murieron, excepto dos que murieron fuera de la UCI antes del alta del hospital, estaban en ventilación mecánica y se encontró una relación directa entre mayor mortalidad y más alto nivel neurológico (Figura 2). La duración media de la ventilación mecánica entre los supervivientes y no supervivientes fue de 12 (rango $=1-98$, RIC $=2-25$ días) y cuatro días (rango $=0-28$, RIC $=2-16$ días), respectivamente. La estancia media hospitalaria fue de 17.5 días (rango = 1-141, RIC = 9-29.5 días).

Seis pacientes al alta (11\%) tenían recuperación completa y $21(39 \%)$ recuperación parcial. Entre los 48 pacientes con ninguna recuperación o recuperación parcial, el estado neurológico más frecuente fue paraplejia en $67 \%(\mathrm{n}=32$; Tabla 2). Cinco (29\%) de 17 pacientes con accidente de tránsito y $18(60 \%)$ de 30 pacientes con herida por arma de fuego no tuvieron recuperación al alta.

\section{Discusión}

Existe una gran variación entre las tasas de TRM en los diferentes países (3), ciudades y aun entre hospitales. Estas diferencias pueden tener varias razones como son el grado de desarrollo económico de los países (1) y su nivel de

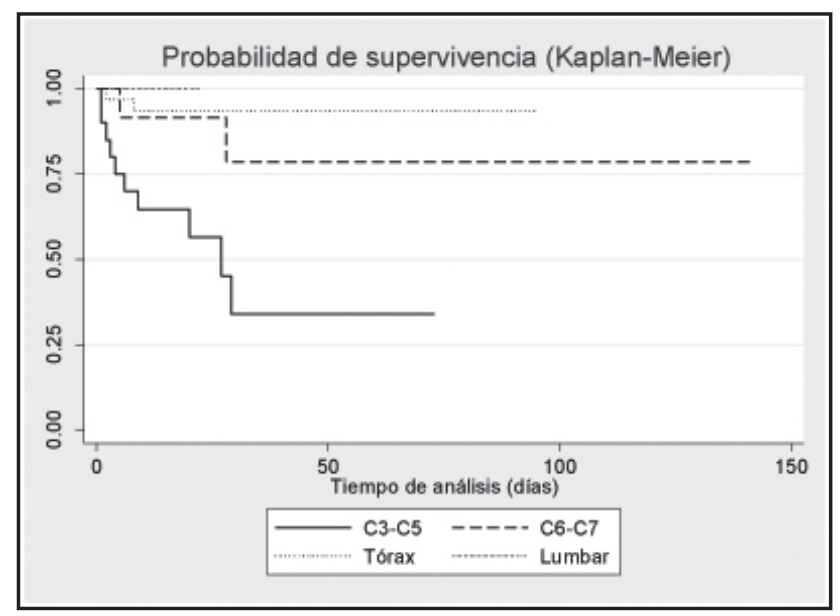

Figura 2. Mortalidad hospitalaria de acuerdo con el nivel de lesión neurológica. Se aprecia mayor mortalidad en los pacientes con niveles más altos de lesión.

Tabla 2. Estado neurológico al alta en 48 pacientes con trauma raquimedular y ninguna recuperación o recuperación parcial.

\begin{tabular}{|l|c|}
\hline Estado neurológico & Número (porcentaje) \\
\hline Paraplejia & $32(67)$ \\
Cuadriparesia & $6(13)$ \\
Cuadriplejia & $4(8)$ \\
Hemiparesia & $2(4)$ \\
Paraparesia & $2(4)$ \\
Paresia de miembros superiores & $2(4)$ \\
\hline
\end{tabular}

violencia (20). Nuestra frecuencia de 12 casos por 1000 pacientes admitidos a la UCI es baja, comparada con otro tipo de pacientes admitidos por enfermedades críticas generales; sin embargo, ésta es comparable con los datos del mundo entero (3-5). Aunque el TRM puede afectar a individuos de cualquier edad, son los adultos en la segunda y tercera décadas, los principalmente afectados (20). Nuestro estudio tuvo una población con edad media de 28 años y la relación hombre/mujer fue de 9 a 1 . Este hallazgo puede ser entendido en el contexto de que la principal causa de TRM fue la violencia, lo cual también puede explicar el alto número de lesiones asociadas como torácicas y en extremidades en 57 y $24 \%$, respectivamente. Recientemente, Sidhu (21) en una revisión sistématica de la literatura acerca del pronóstico y el manejo de los pacientes con TRM secundario a lesión por arma de fuego, encontró que la edad media fue 27 años y el $87 \%$ fueron hombres, hallazgos que concuerdan con los datos encontrados en otros estudios (20-22) al igual que en el nuestro. Blair y colaboradores caracterizaron a los militares que sufrieron lesiones traumáticas espinales en los conflictos de Irak y Afganistán (23). Ellos encontraron una prevalencia de $5.45 \%$ de TRM entre los pacientes con trauma espinal y sólo $15 \%$ fueron secundarios a herida por arma de fuego. El 90\% correspondía a hombres y la edad promedio fue de 26.5 años. Pese a que estos datos son similares a lo encontrado en nuestra investigación, por un lado, la edad y el género están fuertemente influenciados por ser el registro de una población militar, y por el otro, las características de balística son diferentes, lo que no hace comparable los resultados encontrados (21).

Consistente con los resultados de otros estudios (3, 7, $24,25)$ y a pesar de marcadas diferencias con respecto a la etiología, los niveles más frecuentemente afectados fueron el cervical (47\%) y el torácico (47\%). No obstante, en una reciente revisión sistemática de la literatura en donde se incluyeron más de 1000 pacientes civiles con TRM por arma de fuego, el nivel neurológico más frecuentemente afectado fue el torácico (49\%), seguido por el cervical $(30 \%)$ y el lumbosacro (21\%) (21). Nosotros no tuvimos esa misma distribución con respecto al nivel neurológico y esto puede estar relacionado con el grado de violencia y la naturaleza del conflicto en nuestro país. Colombia es un país con una de las tasas de homicidios más altas de la región con 30 casos por 100000 habitantes, que está por encima del promedio de Suramérica de 20 casos (26). Dentro de Colombia y para el año 2013, Medellín tuvo una de las tasas más altas de homicidios del país con 38 casos por 100000 habitantes de acuerdo con el boletín epidemiológico del Instituto Nacional de Medicina Legal (27). La población más afectada fueron los hombres entre 20 y 30 años de edad, con baja escolaridad y bajo estrato socioeconómico. El 76\% de los homicidios fueron causados por armas de fuego y $80 \%$ de los casos ocurrió en áreas urbanas. En nuestro estudio encontramos una distribución similar del TRM secundario a heridas por arma de fuego y el sitio más afectado fue el cervical, lo cual 
nos sugiere la intencionalidad letal del agresor. Previamente Lugo y colaboradores había encontrado datos similares en nuestro medio (25).

Por encima del $50 \%$ de los pacientes de nuestro estudio presentaron complicaciones, tanto infecciosas como no infecciosas, lo cual estuvo por encima de lo encontrado en la revisión realizada por De Barros en Brasil con tan sólo $24 \%$ de lesión de órganos abdominales y $20 \%$ de hemoneumotórax (22). El choque medular (37\%) y las atelectasias (31\%) pueden reflejar el alto porcentaje de pacientes con lesión cervical, dado que las lesiones a este nivel generan choque medular, que es atribuido a la interrupción en las vías autonómicas de la médula espinal, que genera disminución de la resistencia vascular periférica. Por otro lado las atelectasias se pueden relacionar con la debilidad de los músculos respiratorios (músculos intercostales) y el deterioro secundario en la capacidad de depurar las secreciones bronquiales (28).

La media de la estancia hospitalaria luego de sufrir el TRM en nuestra serie fue de 17.5 días. Ésta es comparable con la media de estancia de 16.5 días recientemente publicada en Canadá (4). La ventilación mecánica fue requerida en $56 \%$ de los pacientes y $60 \%$ toleraron la liberación exitosa del soporte ventilatorio. De igual forma con lo reportado en la literatura (29), 60\% de nuestros pacientes fueron liberados exitosamente del soporte ventilatorio. Aunque es aceptado que la traqueostomía puede mejorar la mecánica respiratoria y puede facilitar el desmonte de la ventilación mecánica con la consecuente reducción en la duración del soporte ventilatorio y en los días de estancia en UCI, sólo a 37\% (dos de 14 pacientes se les realizó traqueostomía dentro de la primera semana) se les realizó traqueostomía en nuestra población, una proporción significativamente menor que lo publicado por otros grupos (30).

La duración media de la ventilación mecánica en sobrevivientes y no sobrevivientes fue de 12 y cuatro días respectivamente. Esta duración es marcadamente más corta que la reportada por Ganuzza (30), aunque esto puede ser explicado por una mayor mortalidad en los pacientes con lesión cervical que explicaría el menor tiempo de esta terapia en los pacientes no sobrevivientes. La duración más corta de la ventilación mecánica entre los sobrevivientes no pudimos explicarla de forma adecuada. La mortalidad total de pacientes con TRM en nuestro hospital es más alta que la reportada en otros estudios $(2,4,31,32)$. Este hallazgo puede estar relacionado con la principal etiología (herida por arma de fuego: 57\%) y el alto nivel neurológico comprometido (cervical: 47\%). De hecho, todos los pacientes que murieron requirieron ventilación mecánica y la más alta mortalidad ocurrió en los pacientes con más alto nivel neurológico afectado.

El patrón de recuperación neurológica fue considerado completo en sólo $11 \%$ de los pacientes. Esta tasa no es comparable con otros reportes mundiales y es significativamente menor $(4,7,25)$, aun comparado con estudios de
TRM por heridas por arma de fuego en donde se encontró un déficit completo al final del seguimiento cercano a $30 \%$ $(20,24)$. No obstante, estos son resultados de trabajos retrospectivos en donde el seguimiento no es realizado de forma adecuada.

Lo que está claro es que en general los pacientes con TRM secundario a heridas por arma de fuego tienen déficit completo desde el principio y una menor recuperación que otros tipos de TRM a largo plazo $(20,21,23,25)$. Probablemente esto sea debido a que el proyectil de arma de fuego produce una lesión medular directa y la onda expansiva junto a la cavitación temporal generan destrucción del tejido circundante con afección de niveles superiores e inferiores al nivel de la lesión $(22,33)$.

Cuarenta y ocho pacientes no tuvieron o tuvieron recuperación parcial y el estado neurológico más frecuente al momento del alta fue paraplejia en $67 \%$ de los pacientes. Esto muestra el gran impacto social y económico del TRM en nuestra sociedad. Tres pacientes (8\%) fueron dados de alta a un hospital de cuidado crónico y esto es comparable con lo reportado en Estados Unidos (3). No obstante, estos datos pueden estar subvalorados por el diseño del estudio (retrospectivo) y las características de nuestra población (muchas veces flotante), condiciones que no permiten realizar un seguimiento a largo plazo de los pacientes.

Finalmente, como se esperaba, el peor desenlace fue encontrado en los pacientes con trauma cervical. Comparado con pacientes con TRM con nivel torácico, los pacientes con TRM cervical tenían más larga estancia, más necesidad de soporte ventilatorio, mayor tasa de complicaciones infecciosas y mayor mortalidad.

Este estudio realizado en un hospital de tercer nivel de la ciudad de Medellín fue un intento por caracterizar epidemiológicamente el TRM en nuestro país y adiciona información clínica relevante a estudios previos realizados en nuestro medio (25). Sin embargo, nuestro estudio tuvo algunas limitaciones importantes. Como fue realizado en un solo centro no es posible estimar ni la incidencia ni la prevalencia del TRM en la ciudad. De la misma forma, por el tipo de diseño de nuestro estudio los resultados no pueden ser extrapolados a otras instituciones o ciudades. Finalmente, el estado neurológico y las complicaciones fueron medidas sólo al alta del hospital y esto podría subestimar el impacto real del problema. Aunque por las características de nuestra población el seguimiento posterior al egreso no es fácil debido a que en muchas ocasiones es una población flotante con cambios frecuentes de sitio de residencia e inasistencia a controles posteriores al alta.

\section{Conclusiones}

Esta investigación provee una medida descriptiva de la frecuencia y naturaleza del TRM agudo tratado en un hospital de tercer nivel en países en vía de desarrollo. Aunque el TRM no es una condición frecuente en nuestras unidades de cuidados intensivos, éste representa una patología con un 
impacto grande y refleja el problema de las armas de fuego en nuestra sociedad. El TRM afecta a población masculina joven, su mortalidad, estancia, complicaciones infecciosas y discapacidad tienen relación directa con el nivel de lesión.

\section{Conflictos de interés}

Los autores declaran no tener conflictos de interés en este artículo.

\section{Referencias}

1. Chiu WT, Lin HC, Lam C, Chu SF, Chiang YH, Tsai SH. Review paper: epidemiology of traumatic spinal cord injury: comparisons between developed and developing countries. Asia Pac J Public Health 2012;22(1):9-18.

2. Sayer FT, Kronvall E, Nilsson OG. Methylprednisolone treatment in acute spinal cord injury: the myth challenged through a structured analysis of published literature. The Spine Journal 2006;6(3):335-43.

3. Jackson AB, Dijkers M, Devivo MJ, Poczatek RB. A demographic profile of new traumatic spinal cord injuries: change and stability over 30 years. Arch Phys Med Rehabil. 2004;85(11):1740-8.

4. Pickett GE, Campos-Benitez M, Keller JL, Duggal N. Epidemiology of traumatic spinal cord injury in Canada. Spine (Phila Pa 1976). 2006;31(7):799-805.

5. Silberstein B, Rabinovich S. Epidemiology of spinal cord injuries in Novosibirsk, Russia. Paraplegia. 1995;33(6):322-5.

6. Trinidad E, Hlatky R. Critical care of the patient with traumatic brain and spine injury. In: Irwin RS, Rippe JM, editors. Irwin and Rippe's Intensive Care Medicine. $6^{\text {th }}$, Lippincott Williams \& Wilkins; 2002. pp. 1879-98.

7. Agarwal P, Upadhyay P, Raka K. A demographic profile of traumatic and nontraumatic spinal injury cases: a hospital-based study from India. Spinal Cord. 2007; 45(9): 597-602.

8. DeVivo MJ, Chen Y. Trends in new injuries, prevalent cases, and aging with spinal cord injury. Arch Phys Med Rehabil. 2011; 92(3): 332-8.

9. Esteban A, Anzueto A, Frutos F, Alía I, Brochard L, Stewart TE, et al. Characteristics and outcomes in adult patients receiving mechanical ventilation: a 28-day international study. JAMA. 2002;287(3):345-55.

10. Esteban A, Frutos F, Tobin MJ, Alía I, Solsona JF, Valverdú I, et al. A comparison of four methods of weaning patients from mechanical ventilation. Spanish Lung Failure Collaborative Group. N Engl J Med. 1995; 332(6): 345-50.

11. Blackwood B, Alderdice F, Burns K, Cardwell C, Lavery G, O'Halloran P. Use of weaning protocols for reducing duration of mechanical ventilation in critically ill adult patients: Cochrane systematic review and meta-analysis. BMJ. 2011; 342: 1136-49.

12. Charlifue S, Apple D, Burns SP, Chen D, Cuthbert JP, Donovan WH, et al. Mechanical ventilation, health, and quality of life following spinal cord injury. Arch Phys Med Rehabil. 2011;92(3):457-63.

13. Scheinhorn DJ, Hassenpflug MS, Votto JJ, Chao DC, Epstein SK, Doig GS, et al. Ventilator-dependent survivors of catastrophic illness transferred to 23 longterm care hospitals for weaning from prolonged mechanical ventilation. Chest. 2007;131(1):76-84

14. Hsu CL, Chen KY, Chang CH, Jerng JS, Yu CJ, Yang PC. Timing of tracheostomy as a determinant of weaning success in critically ill patients: a retrospective study. Crit Care. 2005; 9(1): R46-52.

15. MacIntyre NR, Epstein SK, Carson S, Scheinhorn D, Christopher K, Muldoon S, National Association for Medical Direction of Respiratory Care. Management of patients requiring prolonged mechanical ventilation: report of a NAMDRC consensus conference. Chest. 2005;128(6):3937-54

16. DeVivo MJ, Ivie CS. Life expectancy of ventilator-dependent persons with spinal cord injuries. Chest. 2005;108(1): 226-32.

17. Knaus WA, Wagner DP, Draper EA, Zimmerman JE, Bergner M, Bastos PG, et al. The APACHE III prognostic system. Risk prediction of hospital mortality for critically ill hospitalized adults. Chest. 1991; 100(6): 1619-36.

18. American Spinal Injury Association. International standards for neurological classification of spinal cord injury. American Spinal Injury Association. Chicago: ASIA, 2000.

19. Maynard FM Jr, Bracken MB, Creasey G, Ditunno JF Jr, Donovan WH, Ducker TB, et al. International Standards for Neurological and Functional Classification of Spinal Cord Injury. American Spinal Injury Association. Spinal Cord. 1997; 35: 266-74

20. Beaty N, Slavin J, Diaz C, Zeleznick K, Ibrahimi D, Sansur CA. Cervical spine injury from gunshot wounds. J Neurosurg Spine 2014; Jun 13:1-8. [Eoub ahead of print].

21. Sidhu GS, Ghag A, Prokuski V, Vaccaro AR, Radcliff KE. Civilian gunshot injuries of the spinal cord: a systematic review of the current literature. Clin Orthop Relat Res 2013; 471: 3945-55.

22. de Barros Filho TE, Cristante AF, Marcon RM, Ono A, Bilhar R. Gunshot injuries in the spine. Spinal cord 2014; 52: 504-10.

23. Blair JA, Patzkowski JC, Schoenfeld AJ, Cross Rivera JD, Grenier ES, Lehman RA Jr, et al. Spinal column injuries among Americans in the global war on terrorism. J Bone Joint Surg Am 2012; 94(18): e135 (1-9).

24. Tuğcu I, Tok F, Yılmaz B, Göktepe AS, Alaca R, Yazıcıoğlu K, et al. Epidemiologic data of the patients with spinal cord injury: seven years' experience of a single center. Ulus Travma Acil Cerrahi Derg. 2011;17(6):533-8.

25. Lugo LH, García HI. Pérfil epidemiológico de la Lesión Medular en Medellín (Colombia) 1995-1999. Revista Colombiana de Medicina Física y Rehabilitación. 2002; 13: 26-38.

26. Global Study on Homicide 2011. Trends, contexts, data. United Nations Office on Drugs and Crime. http://www.unodc.org/documents/data-and-analysis/statistics/ Homicide/Globa_study_on_homicide_2011_web.pdf.

27. Comportamiento del homicidio, Colombia, 2013. Instituto Nacional de Medicina Legal y Ciencias Forenses. http://www.medicinalegal.gov.co/forensis 1 ;jsessionid =C66D35691D17F9283F2AE304DD58E453.

28. Cotton BA, Pryor JP, Chinwalla I, Wiebe DJ, Reilly PM, Schwab CW. Respiratory complications and mortality risk associated with thoracic spine injury. I Trauma. 2005; 59(6): 1400-7.

29. Call MS, Kutcher ME, Izenberg RA, Singh T, Cohen MJ. Spinal cord injury: outcomes of ventilatory weaning and extubation. J Trauma. 2011;71(6):1673-9.

30. Ganuza JR, Garcia Forcada A, Gambarrutta C, Diez De La Lastra Buigues E, Merlo Gonzalez VE, Paz Fuentes F, et al. Effect of technique and timing of tracheostomy in patients with acute traumatic spinal cord injury undergoing mechanical ventilation. J Spinal Cord Med. 2011;34(1):76-84.

31. Albert T, Ravaud JF. Tetrafigap Group. Rehabilitation of spinal cord injury in France: a nationwide multicentre study of incidence and regional disparities. Spinal Cord. 2005; 43(6): 357-65.

32. Chen HY, Chiu WT, Chen SS, Lee LS, Hung CI, Hung CL, et al. A nationwide epidemiological study of spinal cord injuries in Taiwan from July 1992 to June 1996. Neurol Res 1997;19(6):617-22.

33.Jaiswal M, Mittal RS. Concept of gunshot wound spine. Asian Spine J 2013;7:359-64. 